\title{
A short overview on the use of metabolomic multi-platforms in biomedical research
}

\author{
Álvaro González-Domínguez a,b , Ana Sayago a,b Ángeles Fernández-Recamales a,b, \\ Raúl González-Domínguez (E-mail: raul.gonzalez@dqcm.uhu.es) ${ }^{\text {a,b }}$ \\ ${ }^{a}$ Department of Chemistry, Faculty of Experimental Sciences, University of Huelva. 21007, Spain. \\ ${ }^{b}$ International Campus of Excellence CeiA3, University of Huelva. 21007, Spain.
}

\begin{tabular}{|l|l|}
\hline Graphical Abstract & $\begin{array}{l}\text { Abstract. } \\
\text { The integration of various analytical platforms } \\
\text { is emerging as a very interesting alternative with } \\
\text { the aim to maximize coverage in metabolomics. } \\
\text { In this work, we present a concise review of the } \\
\text { most commonly used metabolomic multi- } \\
\text { platforms in biomedical research. }\end{array}$ \\
\hline
\end{tabular}

Metabolomics is a very interesting option in biomedical research because of its potential to investigate metabolic alterations associated with disease pathogenesis and progression. However, due to the complexity of the human metabolome, metabolomic analysis usually requires the combination of complementary analytical platforms to maximize metabolite coverage.

The integration of reversed-phase liquid chromatography (RP-LC) and gas chromatography (GC) coupled to mass spectrometry (MS) is the most commonly employed alternative, since RP-LC-MS provides a lot of information about the presence of low polar compounds (mainly lipids), while GCMS allows monitoring low molecular weight metabolites [1-3]. On this basis, the combination of reversed-phase and hydrophilic interaction liquid chromatography (HILIC) is also an interesting strategy, since the coupling HILIC-MS allows analyzing polar compounds in biological samples without the recourse of the derivatization step needed in gas chromatography [4-6]. Alternatively, capillary electrophoresis (CE) can also resolve the polar metabolome, but its combination with RP-LCMS has only been proposed in a few works [7-8]. On the other hand, nuclear magnetic resonance (NMR) also complements the analytical performance of previously described techniques based on MS, since NMR can be applied for high-throughput metabolomic fingerprinting of biological samples, prior to in depth analysis by GC-MS, LC-MS or CE-MS [9-11]. In this line, direct mass spectrometry analysis can also be employed for a preliminary metabolic screening, and then complemented with conventional hyphenated approaches [12-13]. 
To conclude, it should be noted that none analytical platform is able to accomplish a global characterization of the metabolome in a single analysis, so the application of metabolomic multiplatforms is necessary. In the author's opinion, an interesting alternative is the combination of direct mass spectrometry analysis, to carry out a "first pass" screening, with RP-LC-MS (focused on lipid compounds) and GC-MS (for the analysis of the low molecular weight fraction of the metabolome).

\section{References}

[1] González-Domínguez et al. Biochim. Biophys. Acta, 2014, 1842, 2395-2402.

[2] Struck-Lewicka et al. J. Pharm. Biomed. Anal., 2015, 111, 351-361.

[3] Lu et al. Diabetologia, 2016, 59, 2349-2359.

[4] Ibañez e al. J. Chromatogr. A, 2013, 1302, 65-71.

[5] Zhang et al. PLoS One, 2013, 8, e65880.

[6] García-Cañaveras et al. J. Proteome Res., 2011, 10, 4825-4834.

[7] Alberice et al. J. Chromatogr. A, 2013, 1318, 163-170.

[8] Balderas et al. Electrophoresis, 2013, 34, 2882-2890.

[9] Chen et al. Sci. Rep., 2014, 4, 5855.

[10] Deng et al. Anal. Chem., 2016, 88, 7975-7983.

[11] Garcia-Perez et al. Anal. Chem., 2010, 82, 203-210.

[12] Lin et al. Analyst, 2010, 135, 2970-2978.

[13] González-Domínguez et al. Electrophoresis, 2017, 38, 2313-2322. 